\title{
Does Backing Up Behavior Explain the Efficacy-Performance Relationship in Teams?
}

Small Group Research 42(4) 458-474

(C) The Author(s) 201 I

Reprints and permission: sagepub.com/journalsPermissions.nav DOI: $10.1177 / 1046496410390964$ http://sgr.sagepub.com ๑SAGE

\section{Christopher O.L.H. Porter', Celile Itir Gogus², and Race Chien-Feng $\mathbf{Y u}^{3}$}

\begin{abstract}
Although the relationship between collective efficacy beliefs and team performance has been well-documented, few studies have explored the causal mechanisms that might explain these effects. In the current study, the authors explore the role of backing up behavior, a specific form of teamwork behavior, in explaining why high efficacy beliefs lead to high levels of team performance. Participants were 416 undergraduate business students who were organized into 104 four-person teams. The teams worked on an interdependent, computerized, decision-making task. Results of the study revealed that collective efficacy beliefs were positively related to team performance and that backing up behaviors largely mediated these effects. The theoretical and practical implications of these findings are discussed.
\end{abstract}

\section{Keywords}

collective efficacy, efficacy-performance, backing up behavior, teamwork, team performance

\footnotetext{
'Texas A\&M University, College Station

${ }^{2}$ Bilkent University, Ankara, Turkey

${ }^{3}$ Ministry of National Defense of the Republic of China-Army, Taipei

\section{Corresponding Author:}

Christopher O.L.H. Porter, Department of Management, Mays Business School, Texas A\&M University, 420 Wehner Building, MS 422I, College Station, TX 77843-422I

Email: cporter@mays.tamu.edu
} 
Collective efficacy is a team's belief that it can successfully perform a specific task (Lindsley, Brass, \& Thomas, 1995). It is a shared belief in a collective's (e.g., team's) capabilities to organize and execute in the course of action (Bandura, 1997). A number of empirical investigations have demonstrated that team efficacy beliefs are positively related to team performance (e.g., Gibson, 1999). Two recent meta-analytic studies have explored the efficacy-performance relationship in teams. Gully, Incalcaterra, Joshi, and Beaubien (2002) provided the first and found strong support for the efficacyperformance relationship $(r=.41)$. More recently, Stajkovic, Lee, and Nyberg (2009) similarly reported evidence of a positive relationship efficacyperformance relationship $(r=.35)$.

Although empirical work such as this has greatly increased our understanding of the efficacy-performance relationship, it is not without two important limitations. The first limitation, and the one most central to the current study, concerns potential mediators of the efficacy-performance relationship. Although efficacy beliefs are associated with higher levels of performance in teams, little research has empirically examined the mechanisms underlying this relationship. In other words, although we can be relatively confident that efficacy beliefs are positively associated with performance in groups and teams, previous research has devoted insufficient attention to explaining why this relationship exists. Theoretically, efficacy beliefs encourage teams to initiate action, lead to higher levels of effort, lead to more persistent effort, and increase the likelihood that teams will continue to put forth effort when collective efforts are unsuccessful (Bandura, 1997; Gully et al., 2002). Gully et al. (2002) proposed that the extent to which teams interpret situations as threats or opportunities might explain the efficacy-performance relationship as well. Despite the number of theoretical explanations that have been offered, it is clear that empirical examinations of potential explanatory variables have not kept pace with the theoretical explanations offered for these effects. This led Gully and his colleagues to call specifically for more research that investigates key mediators of the efficacy-performance relationship.

A second limitation concerns the inability of previous research and, by extension, meta-analytic research findings to rule out the possibility that high levels of task performance lead to higher efficacy beliefs. Stated another way, it is commonly assumed that the nature of the relationship is the reversethat higher efficacy beliefs lead to higher performance-however, as Gully et al. (2002) noted, it is difficult to disentangle issues regarding the direction of causality as it concerns the efficacy-performance relationship. They suggested that true experiments along with studies employing lagged research designs would greatly help to address this problem. 
We address these limitations in the current study by developing and testing hypotheses regarding the role that backing up behavior, a specific type of teamwork process, may play in explaining (or mediating) the effects of efficacy beliefs on performance in teams. Teamwork is "those behaviors that coincide with team members' interacting to achieve desired goals and adapt[ing] to circumstances in order to do so" (McIntyre \& Salas, 1995, p. 33). McIntyre and Salas (1995) observed that not only is teamwork important but it also can make or break team effectiveness. More recently, Marks, Mathieu, and Zaccaro (2001) proposed a taxonomy of team processes in which action processes probably best map onto previous discussions of teamwork (e.g., Dickinson \& McIntyre, 1997; McIntyre \& Salas, 1995). Marks and her colleagues identified two specific action processes, namely backing up behavior and performance monitoring. They further suggested that action processes might explain the effects of team-emergent states like collective efficacy on team effectiveness.

In this study, we focus specifically on backing up behavior as a potential explanation for the efficacy-performance relationship because backing up behavior is believed to be the cornerstone of teamwork (Porter, 2005). Backing up behavior is the discretionary provision of resources and task-related effort to another member of one's team that is intended to help that team member obtain the goals as defined by his or her role (Porter et al., 2003). As such, backing up represents an objective manifestation of the extent to which team members devote physical and cognitive resources to one another in an attempt to help each other achieve their team task objectives (Marks et al., 2001; Porter, Gogus, \& Yu, 2010). As previous research has yielded strong support for the effects of backing up behavior on team performance (Barnes et al., 2008; Porter et al., 2003; Porter et al., 2010), but has failed to systematically explore a broad range of antecedents to backing up behavior in teams, we investigate the possibility that efficacy beliefs may represent an important predictor of the extent to which teams engage in backing up behavior, which in turn predicts team performance.

In the sections below, we report the results of a study in which we examine these effects among a sample of undergraduate students working in fourperson teams on a computerized decision-making task in a laboratory setting. We measured efficacy beliefs shortly after the teams were trained on the task and had some experience working together as a team. We measured backing up behaviors that occurred as the team later worked together on the task. Team performance was determined by how teams ultimately performed on the task and was measured at the conclusion of the task. Although, there are some obvious limitations to one's ability to draw causal inferences from 
cross-lagged relationships (Williams \& Podsakoff, 1989), our use of a lagged research design enabled us to better approximate the potential causal effects of collective efficacy beliefs on backing up behavior and team performance. Our use of a lagged research design also enabled us to explore the extent to which the efficacy-performance relationship could be explained by the degree to which teams engaged in backing up behavior. In doing so, we addressed scholars' recent calls to address the lack of research on the direction of causality in the efficacy-performance relationship (Gully et al., 2002), in addition to calls to investigate team capability beliefs in conjunction with team processes (Hirschfeld \& Bernerth, 2008).

\section{Collective Efficacy, Backing Up Behavior, and Team Performance}

As we noted above, although the positive association between efficacy and expected and actual performance is well documented in the teams literature, few studies go beyond demonstrating the existence of this relationship and attempt to examine the mechanisms through which efficacy impacts performance. Our aim in this article is to move the discussion on the efficacyperformance relationship from how teams feel (i.e., their efficacy beliefs) to how teams actually behave and what they do (i.e., teamwork processes). In this way, we sought to empirically test a model consistent with that recently proposed by DeRue, Hollenbeck, Ilgen, and Feltz (2010) that links efficacy to task processes (in our case, team processes) and then to team effectiveness (see also Marks et al., 2001, who suggested that, among other things, collective efficacy beliefs serve as inputs to subsequent team processes and outcomes).

Previous theory has suggested that efficacy beliefs impact what individuals choose to do as a team, how much effort they put forth, and their staying power when collective efforts are unsuccessful (Bandura, 1997; Stajkovic et al., 2009). Similarly, both Gully et al. (2002) and Stajkovic et al. (2009) proposed that the shared sense of confidence among teams with high collective efficacy helps them persevere in the face of adversity. Although some prior theory suggests that collective efficacy beliefs may have some influence on team performance via the goals that teams set (i.e., Bandura, 1997, found that higher efficacy beliefs lead to higher goals which can in turn lead to higher levels of performance), this further suggests that collective efficacy, although representing a motivational belief, may have its greatest impact on team performance because of the specific behaviors that it engenders among members of a team (e.g., DeRue et al., 2010; Marks et al., 2001). 
Backing up behavior is a function of team members being willing to focus on each other's performances and represents one important way in which members devote their resources to one another (Porter et al., 2010). SmithJentsch, Kraiger, Cannon-Bowers, and Salas (2009) recently suggested that when team members perceive high levels of collective ability, they should be more confident that the team can absorb another member's excess workload (e.g., devote their resources to other members' individual workloads) without it hurting the team's overall performance. Therefore, the higher a team's efficacy beliefs, the more likely it is to devote effort and resources across its members as is the case when team members back each other up.

It is also important to note that backing up behaviors represent a form of adaptive behavior in teams (Porter, 2005). When team members back each other up, they help one another develop job-relevant knowledge and skills that, in the long run, should enable adaptability and have the potential to increase team performance. As teams with high efficacy beliefs persist in their attempts to be successful on their tasks and continue to strive toward goal accomplishment, even when it appears that their previous efforts may have been unsuccessful, we expect that they should be more willing than low-efficacy teams to engage in attempts to help one another to adapt on their tasks.

Unfortunately, scholars are only just beginning to examine the effects of efficacy on team processes such as backing up behaviors (e.g., Chen, Thomas, \& Wallace, 2005; Smith-Jentsch et al., 2009). In one of the first of such studies, Marks (1999) found that efficacy beliefs were related to team coordination processes - namely assertiveness, decision making/mission analysis, adaptability/flexibility, situational awareness, leadership, and communication. Although none of the coordination processes measured in her study directly overlap with backing up behaviors in teams, her findings do lend some support to our expectation that collective efficacy beliefs will be positively associated with backing up behavior. Chen et al. (2005) similarly predicted and found evidence that collective efficacy beliefs predicted team action processes. In their study, four specific action processes were measured including the monitoring of progress toward goals: systems monitoring, team monitoring, coordination, and backing up behavior. Thus, although sparse and not always focused specifically on backing up behavior, there is at least some empirical support for our prediction that efficacy beliefs will influence backing up in teams. This leads to the first of our study hypotheses:

Hypothesis 1: Collective efficacy will be positively related to backing up behavior. 
Our expectation that backing up behavior should be related to team performance is relatively straightforward. Nevertheless, it is an important link to establish prior to developing our third and final prediction that backing up behavior will mediate the efficacy-performance relationship in teams. It is generally assumed that teamwork behaviors facilitate the achievement of collective tasks and consequently increase task performance (Rousseau, Aubé, \& Savoie, 2006). Backing up behavior should generally be positively related to team performance because when team members back up one another they help each other develop role-relevant knowledge and skills. Backing up also helps members correct mistakes that others have made (Porter, 2005). Taken together, backing up behavior enables teams to continuously improve and adapt to the unexpected (Kozlowski \& Ilgen, 2006). Several empirical studies have demonstrated the positive effects of backing up behavior on team performance (e.g., Porter, 2005; Porter et al., 2003), although some more recent work has begun to examine potential boundary conditions for these effects (e.g., Barnes et al., 2008; Porter et al., 2010). For instance, Porter et al. (2010) found that backing up behavior was positively related to team performance when teams had a workload distribution problem, but they also predicted and found that its effects decreased as team members' taskwork skills increased over time. This leads to the following hypothesis:

Hypothesis 2: Backing up behavior will be positively related to team performance.

Of particular interest here is the potential for backing up behavior to mediate the effects of collective efficacy on team performance. Marks et al. (2001) and DeRue et al. (2010) both have proposed team process models that suggest that emergent states such as collective efficacy influence team effectiveness, in part, through more proximal teamwork processes such as backing up behavior. Unfortunately, there are few studies that fully test the input-process-output (IPO) predictions that follow from such models (Ilgen, 1999). Fewer studies have moved beyond examining the role of other motivational variables (e.g., goals and effort) as explanatory mechanisms in the efficacy-performance relationship and examined the mediating role of what teams actually do (i.e., teamwork processes), and no studies focus specifically on backing up behavior as a key mechanism on this relationship outside of the training context. This is problematic given the especially critical role that backing up behavior plays in influencing team performance relative to other forms of action processes. 
Following the aforementioned arguments, we expect that backing up behavior will mediate the efficacy-performance relationship in teams. We predict that teams with high efficacy beliefs will engage in higher levels of backing up behavior, which will in turn lead to higher levels of performance. Some empirical support exists for this prediction, although it is not without some limitations. Chen et al. (2005) reported that collective efficacy beliefs predicted team action processes and that these action processes partially mediated the effects of collective efficacy on adaptive team performance. However, their study was conducted in the context of a training intervention and using only two-person teams, both of which led the researchers to encourage future research to address the limited generalizability of their results. The latter limitation is especially important because backing up behavior does not always simply involve one member helping a second member manage his or her workload (Smith-Jentsch et al., 2009). Moreover, because Chen et al. (2005) measured multiple types of action processes and aggregated these measures to compute an overall action processes variable, it is not clear how much of efficacy-performance relationship can be accounted for by backing up behavior alone. This leads to our third and final study hypothesis:

Hypothesis 3: Backing up behavior will mediate the relationship between collective efficacy and team performance.

\section{Method}

\section{Participants}

Participants were 416 undergraduate business majors enrolled in an introductory management course at a large U.S. southern university. Participation in the study was completely voluntary. All participants received extra course credit in addition to the opportunity to win a US $\$ 100$ cash prize (US $\$ 400$ per team) based on their team's performance.

\section{Research Task}

The research task was a modified version of the Distributed Dynamic Decision-making (DDD) Simulation developed for the Department of Defense. The generic version of the DDD Simulation is a realistic command-and-control context that can portray scenarios from high to low fidelity. In this study, we used a version of the simulation that could be worked on by four-person teams 
of participants with little to no military experience $(n=104)$. The objective of the task was for participants to monitor radar tracks and protect a restricted airspace from enemy tracks. To help them meet this objective, teams were given several resources (i.e., subplatforms) to help them monitor and protect the restricted airspace (i.e., tanks, helicopters, jets, and AWACS reconnaissance planes). These resources were distributed among the teams' members such that each member possessed some specialization that was needed for the team to function successfully as a whole. Specifically, each of the subplatforms had different power levels and capabilities for disabling enemy tracks. Resources were divided among the teams' members such that there were a total of 16 subplatforms in each team and each team member had four of one type of subplatform (i.e., DM1 had four AWACS reconnaissance planes, DM2 had four tanks, DM3 had four helicopters, and DM4 had four jets; see Hollenbeck et al., 2002, for a more complete description). Given this type of team structure and distributed resources, there were high levels of task interdependence among team members as each team member had a somewhat unique capability for helping their team meet its objectives.

\section{Study Procedures}

On entering the laboratory, participants were randomly assigned to a team. Within each team, participants were also randomly assigned to one of four computer stations (i.e., DM1, DM2, etc.). The participants then received both declarative training (e.g., how the task would be scored, what parts of the screen to defend, what subplatforms were capable of disabling what enemy tracks) and procedural training (e.g., how to launch subplatforms, how to identify and disable tracks). After the declarative and procedural training, the participants were given approximately 10 min to practice working on the task together as a team without the help of the experimenter. Overall, the training and practice lasted approximately $75 \mathrm{~min}$, and it was during this time that the participants became familiar with the task, each other, and each other's abilities. It was also during this time that participants gained experience working with each other. Following the training and the practice opportunity, each participant received and completed the collective efficacy measure. Next, the team worked on the 30-min task. Backing up behavior and team task performance were measured during the task. At the end of the experiment, the participants were debriefed and dismissed. 


\section{Measures}

Collective efficacy. Collective efficacy was measured with a seven-item scale adapted from Riggs and Knight's (1994) collective efficacy measure (e.g., "The members of this team have excellent task skills" and "This team is not able to perform as well as it should"). Members responded to this measure after the training but before they worked on the actual task. As the collective efficacy items were based on a referent-shift composition model (Chan, 1998), which assumes that there is some true score for each team and within-team variability represents rater error, we calculated $r_{W G(i)}$ to examine whether there was evidence of sufficient within-team agreement to justify aggregating individual collective efficacy perceptions to the team level. There was, in fact, high levels of within-team agreement on this variable $\left(r_{W G(j)}=.89\right)$.

Team task performance. Team performance was measured with the team's defensive score consistent with the team's goal, which was to defend their restricted zones from enemy tracks. Each team began the simulation with 50,000 points and lost 1 point for each second that any enemy track was in the restricted zones and 2 points for each second that any enemy track was in the highly restricted zones (see Hollenbeck et al., 2002, for more on the task's scoring).

Backing up behavior. During the task, each team member experienced a situation in which the nature and number of tracks entering his or her quadrant exceeded his or her capacity to monitor and disable those tracks. These situations are called waves and the tracks that enter a team member's quadrant during the waves are referred to as wave tracks. Backing up behavior was calculated by the total number of times that team members attacked and cleared enemy wave tracks from quadrants that they were not primarily responsible for protecting.

As an example, take a situation in which one team member (e.g., DM2) is overloaded beyond his or her capacity and so heavily engaged in the task that he or she neglects to ask for help. Another team member (e.g., DM1) may recognize that DM2 needs help but might not be able to devote his or her resources in a way that might directly help DM2. Perhaps, in this situation, DM1 can however help DM2 indirectly by putting out a call for help and by directing DM3 and DM4 in providing direct help for DM2 (e.g., telling them exactly where the help is needed and/or telling them exactly what sort of resources they will need to clear targets from DM2's quadrant). Finally, DM2 must be willing to accept the help that DM3 and DM4 could provide rather than telling these two team members that he or she "has it under control" or 
Table I. Means, Standard Deviations, and Zero-Order Correlations

\begin{tabular}{lrrrrr}
\hline & $M$ & \multicolumn{1}{c}{ SD } & 1 & 2 & 3 \\
\hline I. Team performance & 28195.82 & 3898.06 & & \\
2. Backing up behavior & 11.22 & 3.05 & $.67^{* *}$ & \\
3. Team efficacy & 3.29 & 0.32 & $.22^{*}$ & $.19^{*}$ \\
\hline
\end{tabular}

Note: $n=104$.

$* p<.05 . * * p<.01$.

“doesn't need their help." In this example, backing up occurs as a result of a willingness to receive help, a willingness to provide help, and an ability to coordinate individual efforts. This is just the sort of dynamic interaction that can occur when multiple individuals work together on a highly interdependent team task. Finally, it should be noted that, like task performance, backing up behavior was measured objectively by the computer simulation rather than subjectively by the study participants.

\section{Results}

Table 1 presents the means, standard deviations, and zero-order correlations among the study's variables. As can be seen in the table, collective efficacy was positively related to backing up behavior, $r=.19, p<.05$. This finding lends support for Hypothesis 1 . As can also be seen in the table, backing up behavior was positively related to team performance, $r=.67, p<.01$. This finding lends support for Hypothesis 2. To test Hypothesis 3, we followed the procedure proposed by Baron and Kenny (1986) for testing a mediation model. As our primary interest was the ability of backing up behavior to explain the efficacy-performance relationship, we additionally employed a Sobel (1982) test to determine the indirect effect of collective efficacy beliefs on team performance.

Table 2 presents the results of the hierarchical regression we used to test our prediction that backing up behavior would explain the efficacy-performance relationship. As can be seen in the top half of Table 2, the first part of this test included examining the effect of collective efficacy on team performance (see Step 1). As expected, collective efficacy was positively related to team performance, $\beta=.22, p<.05$, and explained a significant amount of its variance, $R^{2}=.05, F(1,102)=5.15, p<.05$. The second part of this test includes examining the effect of backing up behavior on team performance and, as noted in our test of Hypothesis 2, we found evidence that backing up behavior 
Table 2. Hierarchical Mediated Regression Analyses: Predicting Team Task Performance

\begin{tabular}{llll}
\hline & $\beta$ & $\Delta R^{2}$ & $R^{2}$ \\
\hline Step I. Team efficacy & $.22^{*}$ & $.05^{*}$ & .05 \\
\hline Step I. Backing up behavior & $.67^{* *}$ & $.45^{* *}$ & .45 \\
Step 2. Team efficacy & .09 & .01 & .46 \\
\hline
\end{tabular}

Note: $n=104$. Standardized regression coefficients are reported.

$* p<.05 . * * p<.01$.

was positively related to team performance. The final part of this test (as seen in the bottom half of Table 2), includes examining the effect of collective efficacy on team performance, after controlling for backing up behavior. As can be seen in Step 2, collective efficacy did not have an effect on team performance, after controlling for backing up behavior, $\beta=.09, n s$, although this effect was not completely reduced to zero. This finding lends some support for Hypothesis 3, but it also suggests that backing up behaviors only partially mediates the effects of efficacy on performance in teams.

To further explore the nature of the indirect effect that was implied by Hypothesis 3, we also conducted a Sobel (1982) test to examine to what extent the influence of collective efficacy through backing up behavior on team performance was significant. Results of the Sobel test yielded a significant indirect effect for collective efficacy on team performance through backing up behavior, $z=2.20, p<.05$. Thus, consistent with Hypothesis 3, a meaningful amount of the effect of collective efficacy on team performance is explained by the extent to which teams engage in backing up.

\section{Discussion}

The primary goal of this study was to address scholars' calls for researchers to devote more attention to understanding the mechanisms underlying the well-established finding that collective efficacy beliefs are related to team performance (e.g., Gully et al., 2002; Katz-Navon \& Erez, 2005). Our study did so by examining backing up behavior as a key teamwork process variable that might explain this relationship. By doing so, our study is unique in that rather than linking efficacy beliefs to other motivational variables such as persistence, effort, and goals, we linked efficacy beliefs to a concrete, objective, and well-understood team behavior that is especially critical to team effectiveness (Marks et al., 2001; Porter, 2005; Porter et al., 2003). Moreover, 
although many scholars have developed models of team effectiveness that have relied on IPO frameworks, most empirical studies fail to completely test these models. Ilgen (1999) suggested that the various aspects of IPO models (i.e., the inputs, processes, and outcomes) have generally received unequal attention. In this study, we measured each and found that backing up behavior partially mediates the efficacy-performance relationship in teams. Taken together, our findings help explain not only why efficacy has its positive effects on team performance but also how important backing up behavior is, in particular, in explaining this relationship. Finally, our use of a lagged research design, although not allowing us to draw causal inferences, does allow us to be more confident about the potential direction of causality among our study variables.

Although our study was conducted at the team level and speaks directly to the efficacy-performance relationship in teams, our findings nevertheless suggest both why and how collective efficacy and self-efficacy are distinct constructs. In our study, efficacy beliefs engendered a willingness to engage in backing up behaviors and those behaviors had positive effects on team performance. Backing up behavior explained a significant portion of the efficacyperformance relationship in our teams. However, backing up behavior cannot occur in the absence of a social context such as a team. Thus, our findings suggest that scholars should keep in mind that, although self-efficacy and collective efficacy might represent functionally equivalent constructs (i.e., they may have similar effects on individual and team performance, respectively, through similar motivational influences such as effort and/or goals), self-efficacy probably exerts its influence primarily through cognitive-behavioral processes whereas collective efficacy appears to exert much of its influence primarily through social-behavioral processes (Chen \& Kanfer, 2006; Morgeson \& Hofmann, 1999).

Finally, our study was one of few studies (e.g., Barnes et al., 2008; Porter, 2005; Porter et al., 2003) that has explored antecedents of backing up behavior. Our results indicating that collective efficacy predicts backing up behaviors suggest an important practical implication. Smith-Jentsch et al. (2009) recently forwarded the idea that high collective efficacy beliefs in teams might lead to more backing up behavior in teams because higher levels of efficacy provide team members with confidence that they can engage in backing up toward others without neglecting their own roles and responsibilities so much that their team performance, as a whole, suffers. Our findings lend support to this idea and suggest yet another reason why high efficacy beliefs can be functional and should be encouraged in teams. As backing up behavior requires team members to be willing not only to provide help to 
other members of their teams but also to accept help from other members of their teams (Porter et al., 2003), we would additionally submit that high collective efficacy beliefs might instill confidence in team members that attempts by other members of their team to help and assist them will be effective and therefore should be accepted. Extending this idea, an interesting area for future research would be to explore situations in which, although overall collective efficacy beliefs are high, members have lower amounts of confidence in some subset of members in their team. Situations such as this might attenuate the positive effects of collective efficacy on backing up behavior in teams. Somewhat related, another clear avenue for future research would be to explore potential boundary conditions of the positive effects we found for collective efficacy beliefs on backing up behavior.

Although we found support for our study's hypotheses, our study, was not without some limitations that deserve mention. Our study was conducted in a laboratory setting with undergraduate students serving as participants, thus raising the obvious concerns regarding the generalizability of our results. Having said that, we believe that the setting and participants used in this study do not represent a major limitation for several reasons. First, the task we used was an engaging one in which there were high levels of both task and outcome interdependence. Second, although our student participants lacked actual military experience (at least in most cases), our teams had performancebased rewards; had to make decisions under time pressure and threat; and the task required members to interact, participate, and coordinate their inputs to be successful. In this way, our teams could be thought of as tactical decisionmaking teams (McIntyre \& Salas, 1995) or project teams (Ellis et al., 2003) and were similar to a number of teams that exist in actual work organizations (e.g., hospital emergency room teams, managerial teams, police teams, airline crews). Third, one important benefit of using a laboratory setting for conducting our study was our ability to obtain a sufficient sample size for testing our model.

Another limitation is our exclusive focus on backing up behaviors. Teamwork is a multidimensional construct (e.g., McIntyre \& Salas, 1995; Rousseau et al., 2006), but we focused on backing up behavior because previous research has suggested that it is perhaps the most important aspect of teamwork, we wanted to determine how much of the efficacy-performance relationship could be explained by backing up behavior, and we could measure it objectively. Now that we have established that backing up behavior can account for a large portion of the efficacy-performance relationship, researchers should incorporate other teamwork processes into future studies. Given our findings, we suggest that future research continue to focus specifically on 
action teamwork processes (e.g., Marks et al., 2001). In addition, we suggest that researchers empirically distinguish between and account for each processes' independent ability to explain the efficacy-performance relationship.

Despite these limitations, our study has a number of strengths that also deserve mention. First, our design allowed us to address the causal direction problem that plagues much of the empirical work on the efficacy-performance relationship. Gully et al. (2002) suggested that one reason for this problem lies in the fact that most studies are not true experiments, nor do they use lagged designs, but rather are cross-sectional in design. In our study, participants completed the team efficacy measure after they were trained and practiced on the task but prior to the actual task. As efficacy beliefs were formed and measured prior to the performance opportunity, we can conclude the direction of causality with some certainty.

A second strength of our study, and to a large extent a benefit of our use of a laboratory setting, was our ability to objectively measure both backing up behavior and performance. We measured collective efficacy via a selfreport survey measure before participants actually completed the task. Therefore, our study design allows us to rule out common method bias as an explanation for our results. We believe that designs such as this one are particularly important for team researchers as it is likely that social desirability may play some role in how team members respond to questions about the extent to which they actually worked together as a team.

\section{Acknowledgment}

The authors thank Jenny Keng, Joe Martin, and Thomas Lopez for their help collecting data.

\section{Authors' Note}

A previous version of this manuscript was presented at the 65th Annual Meeting of the Academy of Management, Honolulu, Hawaii.

\section{Declaration of Conflicting Interests}

The author(s) declared no potential conflicts of interest with respect to the authorship and/or publication of this article.

\section{Funding}

The author(s) disclosed that they received the following support for their research and/or authorship of this article: This research was supported, in part, by a Mays Business School Summer Research Grant and Grant N00014-96-1-0983 from the Cognitive and Neural Sciences Division of the Office of Naval Research obtained by 
Daniel R. Ilgen and John R. Hollenbeck (Principal Investigators) at Michigan State University. Although support for this work is gratefully acknowledged, the ideas expressed herein are those of the authors and not necessarily endorsed by the funding agency.

\section{References}

Bandura, A. (1997). Self-efficacy: The exercise of control. New York, NY: W. H. Freeman.

Barnes, C. M., Hollenbeck, J. R., Wagner, D. T., DeRue, D. S., Nahrgang, J. D., \& Schwind, K. M. (2008). Harmful help: The costs of backing-up behavior in teams. Journal of Applied Psychology, 93, 529-539. doi:10.1037/0021-9010.93.3.529

Baron, R. M., \& Kenny, D. A. (1986). The moderator-mediator variable distinction in social psychological research: Conceptual, strategic, and statistical considerations. Journal of Personality and Social Psychology, 51, 1173-1182. doi:10.1037/00223514.51.6.1173

Chan, D. (1998). Functional relationships among constructs in the same content domain at different levels of analysis: A typology of composition models. Journal of Applied Psychology, 83, 234-246. doi:10.1037/0021-9010.83.2.234

Chen, G., \& Kanfer, R. (2006). Toward a systems theory of motivated work behavior in teams. Research in Organizational Behavior, 27, 223-267. doi:10.1016/S01913085(06)27006-0

Chen, G., Thomas, B., \& Wallace, J. C. (2005). A multilevel examination of the relationships among training outcomes, mediating regulatory processes, and adaptive performance. Journal of Applied Psychology, 90, 827-841. doi:10.1037/ 0021-9010.90.5.827

DeRue, D. S., Hollenbeck, J. R., Ilgen, D. R., \& Feltz, D. (2010). Efficacy dispersion in teams: Moving beyond agreement and aggregation. Personnel Psychology, 63, 1-40. doi:10.1111/j.1744-6570.2009.01161.x

Dickinson, T. L., \& McIntyre, R. M. (1997). A conceptual framework for teamwork measurement. In M. T. Brannick, E. Salas, \& C. Prince (Eds.), Team performance assessment and measurement (pp. 19-43). Mahwah, NJ: Lawrence Erlbaum.

Ellis, A. P. J., Hollenbeck, J. R., Ilgen, D. R., Porter, C. O. L. H., West, B. J., \& Moon, H. (2003). Team learning: Collectively connecting the dots. Journal of Applied Psychology, 88, 821-835. doi:10.1037/0021-9010.88.5.821

Gibson, C. B. (1999). Do they do what they believe they can? Group efficacy and group effectiveness across tasks and cultures. Academy of Management Journal, 42, 138-152. doi:10.2307/257089

Gully, S. M., Incalcaterra, K. A., Joshi, A., \& Beaubien, J. M. (2002). A meta-analysis of team-efficacy, potency, and performance: Interdependence and level of analysis 
as moderators of observed relationships. Journal of Applied Psychology, 87, 819-832. doi:10.1037/0021-9010.87.5.819

Hirschfeld, R. R., \& Bernerth, J. B. (2008). Mental efficacy and physical efficacy at the team level: Inputs and outcomes among newly formed action teams. Journal of Applied Psychology, 93, 1429-1437. doi:10.1037/a0012574

Hollenbeck, J. R., Moon, H., Ellis, A. P. J., West, B. J., Ilgen, D. R., Sheppard, L., . . Wagner, J. A., III. (2002). Structural contingency theory and individual differences: Examination of external and internal person-team fit. Journal of Applied Psychology, 87, 599-606. doi:10.1037/0021-9010.87.3.599

Ilgen, D. R. (1999). Teams embedded in organizations: Some implications. American Psychologist, 54, 129-139. doi:10.1037/0003-066X.54.2.129

Katz-Navon, T. Y., \& Erez, M. (2005). When collective- and self-efficacy affect team performance: The role of task interdependence. Small Group Research, 36, 437-465. doi:10.1177/1046496405275233

Kozlowski, S. W. J., \& Ilgen, D. R. (2006). Enhancing the effectiveness of work groups and teams. Psychological Sciences in the Public Interest, 7, 77-124.

Lindsley, D. H., Brass, D. J., \& Thomas, J. B. (1995). Efficacy-performance spirals: A multilevel perspective. Academy of Management Review, 20, 645-678. doi:10.2307/258790

Marks, M. A. (1999). A test of the impact of collective efficacy in routine and novel performance environments. Human Performance, 12, 295-309.

Marks, M. A., Mathieu, J. E., \& Zaccaro, S. J. (2001). A temporally based framework and taxonomy of team processes. Academy of Management Review, 26, 356-376. doi: $10.2307 / 259182$

McIntyre, R. M., \& Salas, E. (1995). Measuring and managing for team performance: Lessons from complex environments. In R. A. Guzzo \& E. Salas (Eds.), Team effectiveness and decision-making in organizations (pp. 9-45). San Francisco, CA: Jossey-Bass.

Morgeson, F. P., \& Hofmann, D. A. (1999). The structure and function of collective constructs: Implications for multilevel research and theory development. Academy of Management Review, 24, 249-265. doi:10.2307/259081

Porter, C. O. L. H. (2005). Goal orientation: Effects on backing up behavior, performance, efficacy, and commitment in teams. Journal of Applied Psychology, 90, 811-818. doi:10.1037/0021-9010.90.4.811

Porter, C. O. L. H., Gogus, C. I., \& Yu, R. C. (2010). When does teamwork translate into improved team performance? A resource allocation perspective. Small Group Research, 41, 221-248. doi:10.1177/1046496409356319

Porter, C. O. L. H., Hollenbeck, J. R., Ilgen, D. R., Ellis, A. P. J., West, B. J., \& Moon, H. (2003). Backing up behaviors in teams: The role of personality and 
legitimacy. Journal of Applied Psychology, 88, 391-403. doi:10.1037/00219010.88.3.391

Riggs, M. L., \& Knight, P. A. (1994). The impact of perceived group success-failure on motivational beliefs and attitudes: A causal model. Journal of Applied Psychology, 79, 755-766. doi:10.1037/0021-9010.79.5.755

Rousseau, V., Aubé, C., \& Savoie, A. (2006). Teamwork behaviors: A review and an integration of frameworks. Small Group Research, 37, 540-570. doi:10.1177/ 1046496406293125

Smith-Jentsch, K. A., Kraiger, K., Cannon-Bowers, J. A., \& Salas, E. (2009). Do familiar teammates request and accept more backup? Transactive memory in air traffic control. Human Factors, 51, 181-192. doi:10.1177/0018720809335367

Sobel, M. E. (1982). Asymptotic confidence intervals for indirect effects in structural equation models. In S. Leinhardt (Ed.), Sociological methodology 1982 (pp. 290-312). Washington, DC: American Sociological Association.

Stajkovic, A. D., Lee, D., \& Nyberg, A. J. (2009). Collective efficacy, group potency, and group performance: Meta-analyses of their relationships, and test of a mediation model. Journal of Applied Psychology, 94, 814-828. doi:10.1037/a0015659

Williams, L. J., \& Podsakoff, P. M. (1989). Longitudinal field methods for study reciprocal relationships in organizational behavior research: Toward improved causal analysis. In B. M. Staw \& L. L. Cummings (Eds.), Research in organizational behavior (Vol. 11, pp. 77-140). Greenwich, CT: JAI.

\section{Bios}

Christopher O.L.H. Porter is an associate professor of management at Mays Business School at Texas A\&M University, College Station. He received his PhD in business administration from Michigan State University. His research interests include team leadership, team composition, teamwork processes, feedback interventions, and police performance management.

Celile Itir Gogus is an assistant professor of management in the Faculty of Business Administration at Bilkent University, Ankara, Turkey. She received her PhD in management from Mays Business School at Texas A\&M University. Her research interests include teamwork processes, organizational justice, and family businesses.

Race Chien-Feng $\mathbf{Y u}$ is a professional army officer and is currently a colonel working at the Ministry of National Defense of the Republic of China-Army. He received his $\mathrm{PhD}$ in management from Mays Business School at Texas A\&M University. His research interests include personality theory, leadership, and team effectiveness. 